\title{
Design and Implementation of Arduino based Riders Safe Guard 2.0
}

\author{
K.Murali Chandra Babu, P.A.HarshaVardhini, N.Koteswaramma
}

\begin{abstract}
No matter how careful and attentive people are on road, accidents do happen. The only way to have a safe journey is by following safety measures. To decrease the death rate of bike riders, this paper proposes a solution called Rider's Safe Guard 2.0. Few incidents due to wrong decisions on road may cost our precious life due to recklessness. This work automates the process and the responsibility of decision making is given to the micro controller. Microcontroller makes sure whether the rider is wearing safety gear such as the helmet and whether the ride is under any alcohol intake. By analyzing both these condition the motor starts running and the circuit gets connected to the vehicle ignition switch. Micro controller Arduino Nano and Uno based system insists the rider to wear helmet to ride the bike as long as the motor is on. As there is no declination in road accidents, this system can be made mandatory in every two wheeler and the same can be extended for fourwheelers for wearing seat belts and doesnot allow if the rider is alcoholic.
\end{abstract}

\section{INTRODUCTION}

A total of 17.9 million two-wheelers were sold in our country last year alone, that's a little over 48,000 units everyday. That's a bright sign of development, but on the other side in our country over $1,47,913$ people were killed in road accidents last year, that is more than the number of people killed in all our wars put together. Driving at high speeds, under the influence of alcohol or drugs, tiredness or without a helmet are indeed responsible for higher number of deaths in road crashes. Drunken driving is one of the leading causes of road fatalities. $98.6 \%$ of bikers who died didn't wear a helmet. 1214 road crashes occur every day in India. No matter how careful and attentive may be the rider accidents do happen. The only way to have a safe journey is by following safety measures. In order to reduce the impact of our decision reflexes on our safety, we are letting the machine to take up the responsibility of our safety, when we are riding a two wheeler. On a whole, increasing the safety of the riders is what we are working on. This work develops a system, where it checks whether the rider is wearing the helmet or not and monitors the alcohol consumption level. The system allows the rider to access the bike only when he is equipped by the rider's safe guard 2.0 and satisfying its requirements in real-time.

To design this rider's safeguard 2.0, we need the system to sense the safety of the rider with the helmet and is not drunk in order to ensure whether the rider should be allowed to ride the bike or not. To get the sensors capacitive switch and alcohol sensor working together, we run an algorithm written in Arduino IDE software on an Arduinoboard(Nano).

In recent years many developments and works to overcome these problems.

Ignition interlock systems with multiple alcohol sensors Vehicle

A vehicle ignition interlock system includes a breath analyzer and also controller operably connected to the breath analyzer and to an ignition system of the vehicle. The breath analyzer detects breath alcohol level of a vehicle operator and prevents vehicle ignition if a breath alcohol level is greater than or equal to a threshold value. The controller requires the vehicle operator to periodically take breath analyzer "retests" after vehicle ignition in order to allow vehicle operation to continue. Here the person should take regular retests which can be considered as a draw back.

Breathanalyzer for use in automobile ignition locking systems

An improved breath analyzing apparatus for use in an automobile ignition locking system. A breath sample tube receives a breath sample from a user. The breath sample tube is divided into a high pressure section and a low pressure section by an air flow restrictor. A pressure sensor is connected to the high pressure section to determine when a user is blowing into the breath analyzing apparatus. A temperature sensor measures the temperature of the breath sample to insure that it is the same temperature as human breath. A micropump propels a specified volume of the breath sample into a fuel cell which is used to determine the alcohol content. Control means converts the output of the fuel cell into a reading which represents the breath alcohol content of the breath sample. Heating elements warm the fuel cell when the ambient temperature drops below a specified level. The output of the fuel cell is adjusted based on the time it takes the fuel cell to reach its maximum output. A housing holds all the major components of the breath analyzing apparatus and contains a tamper switch therein which detects when the case is opened. A display on the housing shows the alcohol content of the breath sample as well as displaying instructions and messages for the user The breath analyzer apparatus is used as part of an overall

Revised Manuscript Received on October 15, 2019.

K.Murali Chandra Babu, Department of ECE, Vignan Institute of Technology and Science, Deshmukhi, Telangana, India.

P.A.HarshaVardhini, Department of ECE, Vignan Institute of Technology and Science, Deshmukhi, Telangana, India.

N.Koteswaramma, Department of ECE, Vignan Institute of Technology and Science, Deshmukhi, Telangana, India. 
automobile ignition locking system which prohibits starting requires rolling retests to insure that the driver is still sober.

Non-invasive optical blood alcohol concentration reader and vehicle ignition interlock system and method

A vehicle ignition interlock system including a noninvasive reader of a person's blood-alcohol concentration in combination with ignition interlock circuitry that prevents operation of a vehicle by an intoxicated person. The noninvasive blood-alcohol concentration reader, termed alcohmeter, utilizes optical spectroscopic electromagnetic radiation technology to determine the alcohol levels in the blood. The alcoh-meter is preferably a dash mounted sensor for receiving a person's finger and absorbing incident light from a multiple wavelength light source and causing a light absorption reading to be generated based on the person's blood alcohol concentration in the finger tissue. After registering a reading, the results are compared electronically against a table of impaired/non-impaired levels of blood alcohol concentration. The impaired/non-impaired results are communicated to interlock circuitry that either enables, or disables start-up of the vehicle. If an impaired status is determined, the results are displayed instructing the operator to wait, or find a non-impaired operator. As the system designed is invasive the blood alcohol reading may not be accurate. By keeping the flaws of the above systems in mind we came up with Riders safeguard 2.0.

In order to decrease the death rates, the governments of different states in our country came with numerous rules and regulations such as:

$>\quad$ By enforcing regular checkpoints on the roads.

$>\quad$ By making the helmets compulsory for the riders.

$>\quad$ By using traffic monitoring cameras to strictly punish the law breakers

$>\quad$ By conducting strict drunk and drive checks.

$>\quad$ By resorting to extreme measures like huge fines,

By continuously revising and updating the laws with the rising accident rates.

Even though the above rules were implemented, there has been no significant decline in the accident rates and deaths every year. In fact with all the above rules, the death rates are actually on the rise, everyday more than 1214 people are seriously injured due to bike accidents in our country according to the 2018 survey by The Times Of India.

\section{DESIGN - TRANSMITTER AND RECEIVER SECTION}

The rider safeguard 2.0 system basically consists of two modules:

I. The transmitter section in the helmet

II. The receiver section in the bike

\section{Transmitter section}

The transmitter section has Arduino Uno as the controlling device, connected with two sensors and the wireless transceiver module. Two different types of sensors are used here : the car when the operator is intoxicating. The system also suspending license and imprisonment.

1. The capacitive switch, to sense the presence of the helmet over the riders head.

2. Alcohol sensor, to estimate the Blood Alcohol Concentration level, indirectly by measuring the amount of alcohol in rider's breath.

\section{Sensors}

The touch sensor and the alcohol sensor are placed inside the helmet, together they indicate whether the person is wearing the helmet or not and he is drunk or not. Then the Arduino Nano process the data received from the sensors following an algorithm, transmit a message to the other module in the bike section.

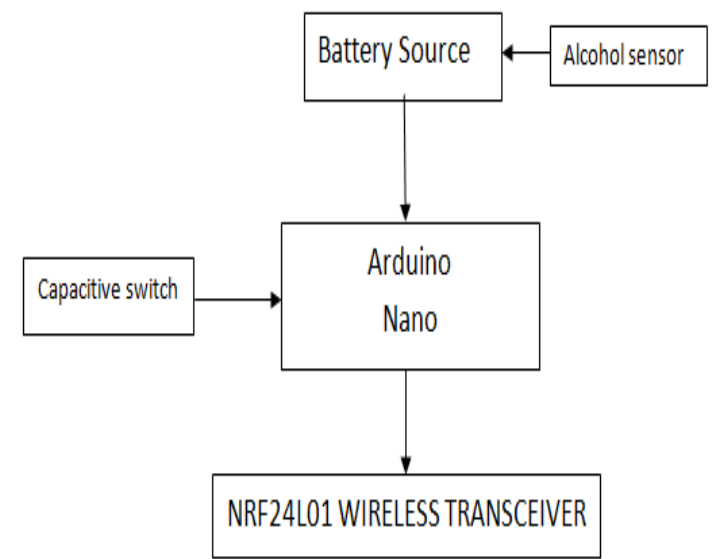

Fig.1. Circuit Diagram of Transmitter section

Receiver section

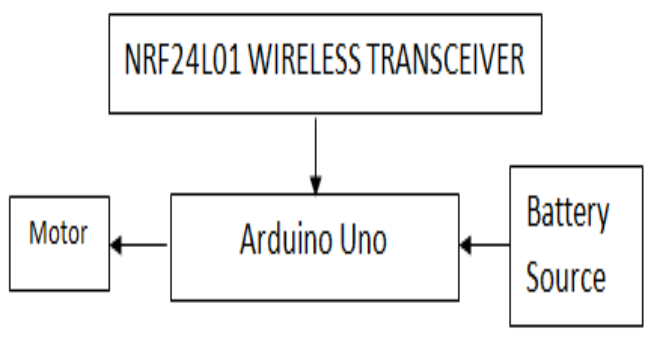

Fig.2. Circuit Diagram of Receiver section

The receiver section has an Arduino Uno which is connected with the wireless transceiver NRF24L01 and controls the operation of the DC motor, depending on the message received from the transmitting module NRF24L01 which is present in the helmet.

\section{Operation of the Motor}

Here the algorithm is written in such a way that the transceiver module constantly listens for the message from the transmitter. Whenever the receiver section identifies a change in the message, it changes the operation of the D.C motor accordingly. The D.C motor has two modes of operation either ON or OFF, which resembles the state

\section{Helmet}

Once the Arduino Nano is powered up, the capacitive switch which uses the capacitance of the electrons from our

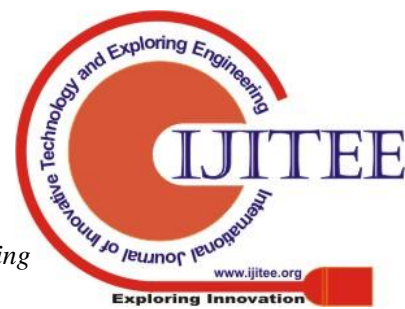


skin to detect whether the rider is wearing the helmet or not and sends a digital signal to the arduino, the arduino checks the alcohol level in the rider's breath using the alcohol/gas sensor MQ3 only if the output of the capacitive switch is positive i.e the rider is wearing the helmet. The alcohol level is measured by the MQ3 sensor and an analog signal is sent to the arduino, now the arduino compares the obtained value with the predefined threshold value in the algorithm and if the value is less than the threshold value, it transmits a signal " $1 "$ else it transmits " 0 " using the NRF24L01 wireless module.
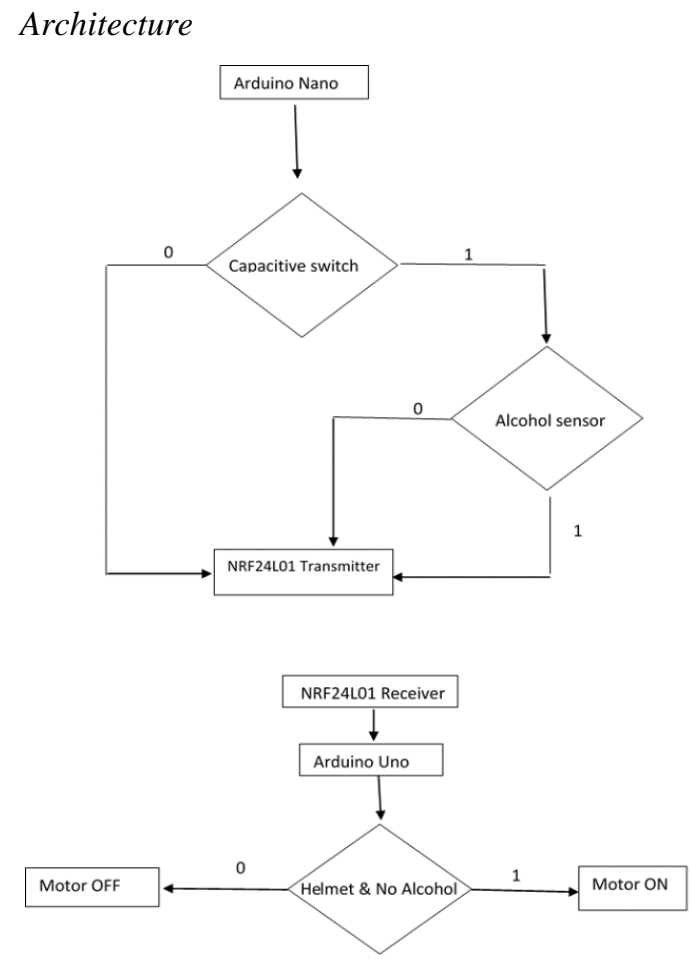

Fig 3. Workflow

\section{Helmet}

Once the Arduino Nano is powered up, the capacitive switch which uses the capacitance of the electrons from our skin to detect whether the rider is wearing the helmet or not and sends a digital signal to the arduino, the arduino checks the alcohol level in the rider's breath using the alcohol/gas sensor MQ3 only if the output of the capacitive switch is positive i.e the rider is wearing the helmet. The alcohol level is measured by the MQ3 sensor and an analog signal is sent to the arduino, now the arduino compares the obtained value with the predefined threshold value in the algorithm and if the value is less than the threshold value, it transmits a signal " 1 " else it transmits " 0 " using the NRF24L01 wireless module.

\section{Motor}

The Arduino Uno gets powered on as soon as the bike battery is turned on and it constantly keeps the radio on i.e the NRF24L01 wireless transceiver module, when the arduinouno receives " 1 " from the transmitter section it turns the motor ON else it switches the motor OFF.

\section{Transmitter Module}

On a whole the transmitter module is all about collecting data from the sensors and analyzing the data to decide whether it is safe for the person to ride the bike or not. On the other hand the receiver section is all about controlling the state of the bike as decided by the transmitter section.

\section{IMPLEMENTATION}

The Arduino Nano is the driving element of all the sensors in the transmitter section. It controls the functioning of the other elements, gathers the data from them, processes the data and also transmits it to the receiver section. The Arduino acts like the brain of the entire circuit. Now let's see how the other components are configured to the Arduinonano on the transmitter section.

\section{Pin Configuration of the transmitter section}

\section{Battery}

The Arduino and the other components are powered by the battery source, which is connected to the Vin(+ve terminal of battery) and GND of the Arduino respectively

\section{Capacitive switch}

The capacitive switch is powered by connecting the Vecto the common Vec of the transmitter circuit andGND pin to the common GND of the circuit. The digital data out pin of the capacitive switch is connected to the DIGITAL pin D3.

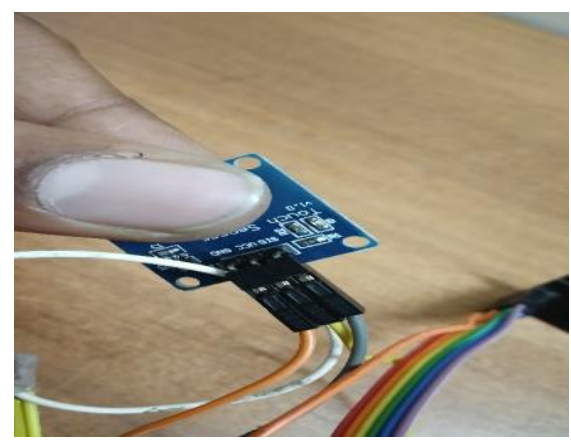

Fig 4.Capacitive switch

\section{Alcohol Sensor}

The alcohol sensor is powered by connecting the Vccto the common Vcc of the transmitter circuit and GND pin to the common GND of the circuit. The analog data out pin of the alcohol sensor is connected to the ANALOG pin A0.

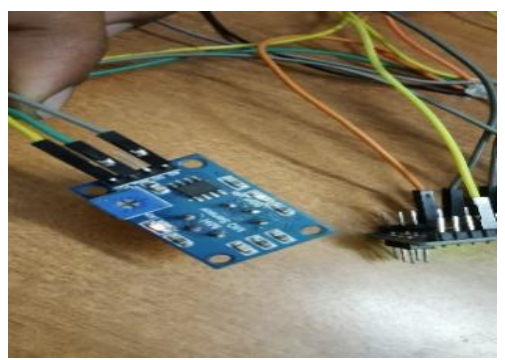

Fig.4. Alcohol Sensor 


\section{NRF24L01}

The NRF24L01 wireless module of the transmitter section is connected to the Arduino Nano by connecting the following respective pins of both boards.

\begin{tabular}{|c|c|c|}
\hline S.NO & PINS OF ARDUINO & PINS OF NRF24L01 \\
\hline 1 & $3.3 \mathrm{~V}$ & VCC \\
\hline 2 & Common GND & GND \\
\hline 3 & 7 & CE \\
\hline 4 & 8 & CSN \\
\hline 5 & 11 & MOSI \\
\hline 6 & 12 & MISO \\
\hline 7 & 13 & SCK \\
\hline
\end{tabular}

Table 1. Pin configuration of the NRF24L01 connected with the Arduino NANO/UNO

The common ground of the entire circuit is connected to the remaining GND pin on the Arduino board and the common Vcc of the entire circuit is connected to the $5 \mathrm{~V}$ pin of the Arduino board.

\section{Receiver Configuration}

Arduinouno controls the operation of the motor, which we show to mimic the operational state of the bike.

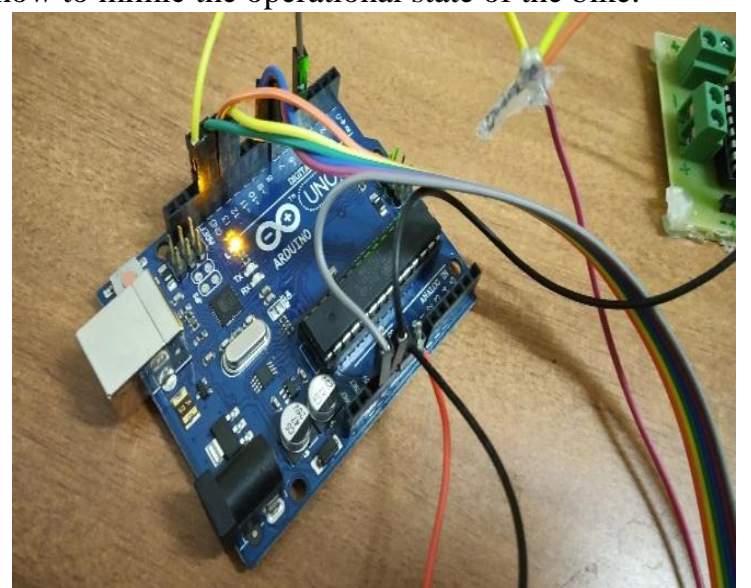

Fig.5. Arduino Uno

Battery

The Arduinouno is powered by the battery source, wherein the +ve terminal of the battery goes to the Vin of the Arduino board and the -ve terminal of the battery goes to the GND of the Arduino board.

\section{NRF24LO1}

The NRF24L01 is connected to the Arduino Uno by following the same connections as provided in the Table. 1

\section{D.C.Motor}

The DC motor is connected to Arduino using the motor driver IC L293D.The controller input to the motor from the Arduino is sent from the DIGITAL pin D4.

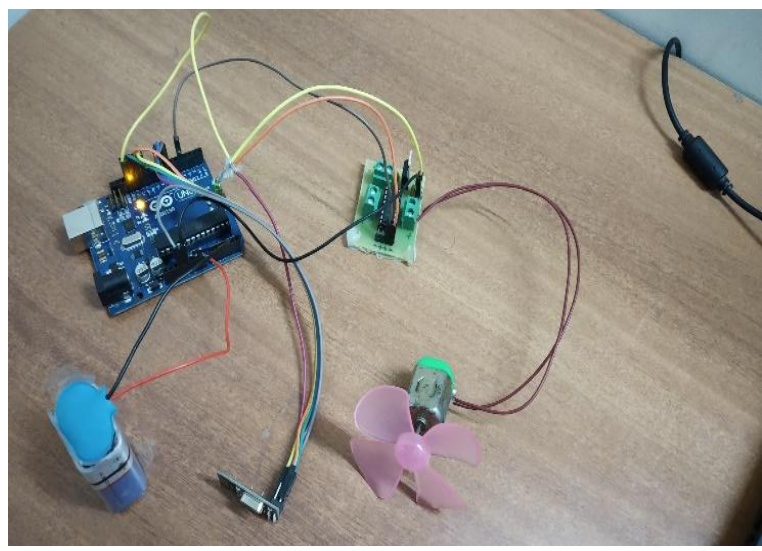

Fig6. Receiver section

Programming Arduino:

Step 1: Downloading the Arduino environment.

We downloaded and installed the latest version of the Arduino IDE from the official website and it is opensource.

Step 2: Installing the required libraries and driver files

For our necessity we installed the RF24.h and nRF24L01.h libraries as we used for the smooth operation of the NRF24L01 wireless module. Also the drivers are updated.

Step 3: Launch the Arduino application, write the code on the editor and save it.

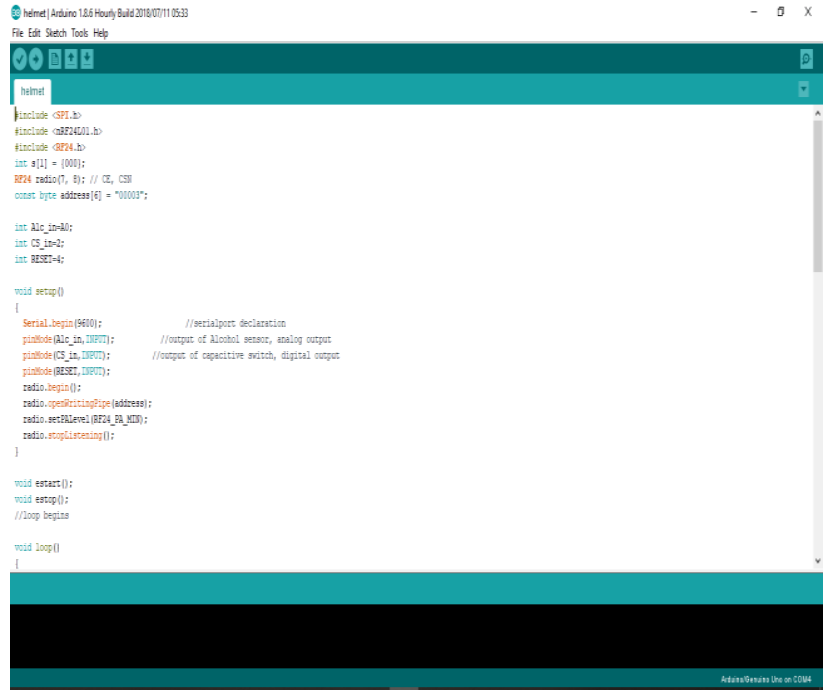

Fig.7. Editor

Step 4: Connect the Arduino:

The Arduino UNO/NANO is connected to the PC via USB cable and select the same from the TOOLS tab.

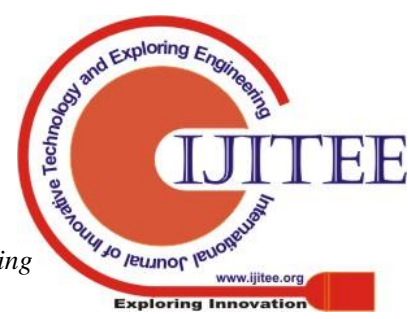




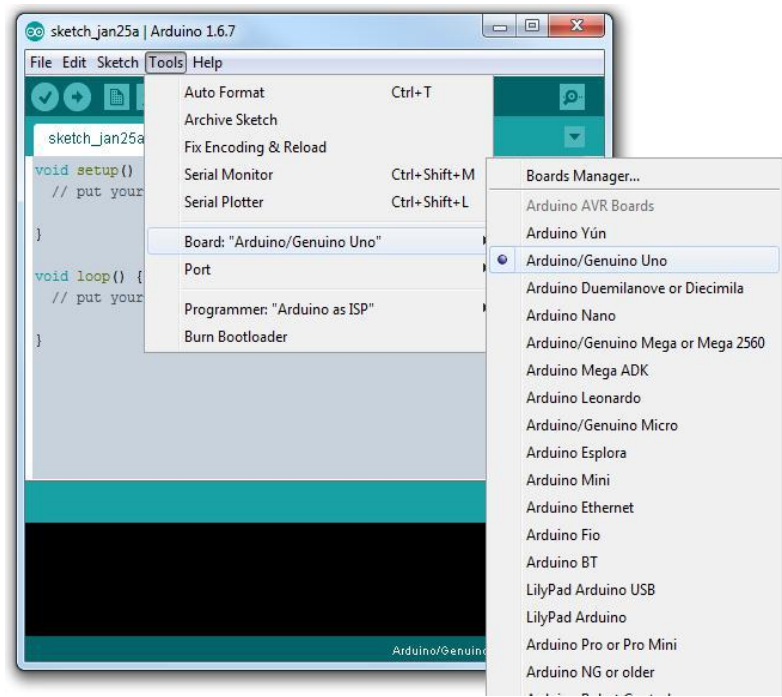

Fig.8. Connecting the arduino

Step 5:Select your Serial port.

Select the serial device of the Arduino board from the Tools | Serial Port menu.

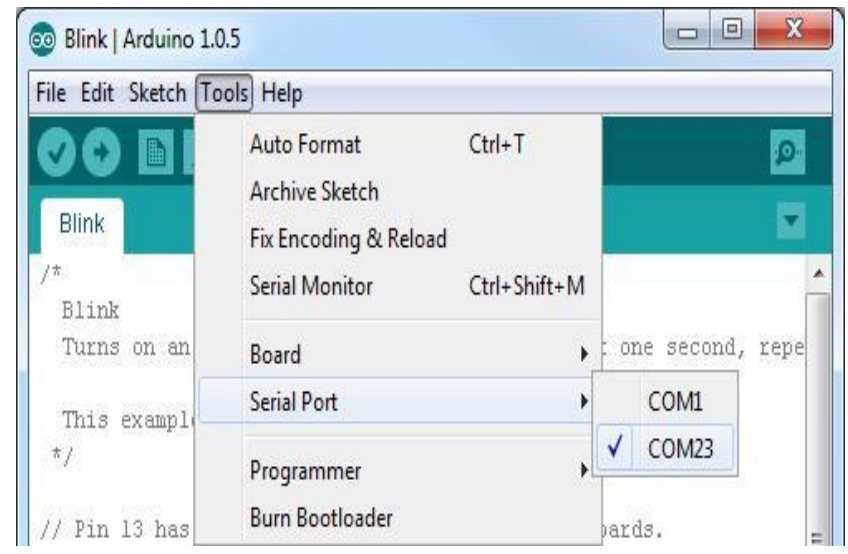

Fig 9. Serial Port

Step 6: Compile the program to know whether there are any errors in the program and if you get any errors debugthem to proceed.

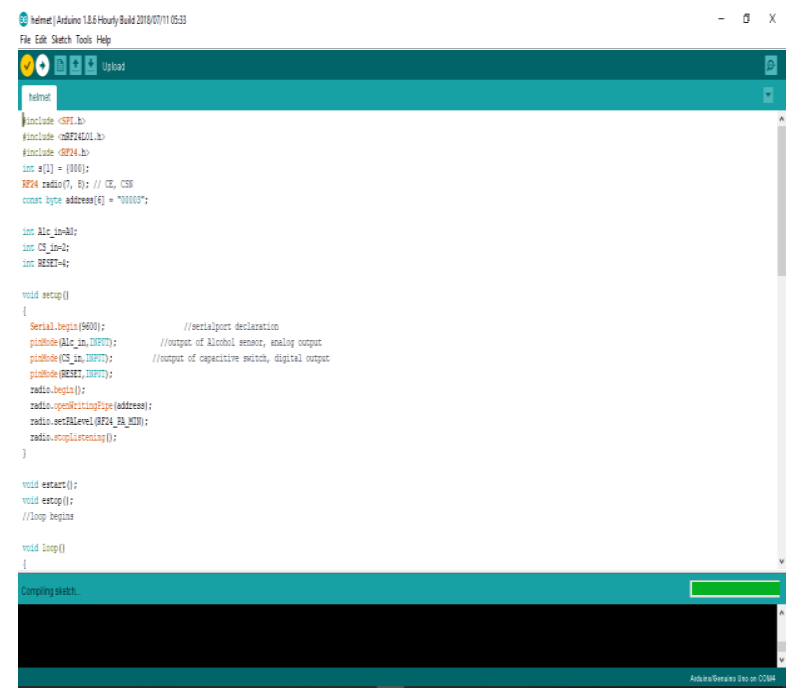

Fig.10. Compilation
Step 7:Upload the program.

Now, simply click the "Upload" button in the environment. Wait a few seconds - you should see the RX and TX led's on the board flashing. If the upload is successful, the message "Done uploading." will appear in the status bar.

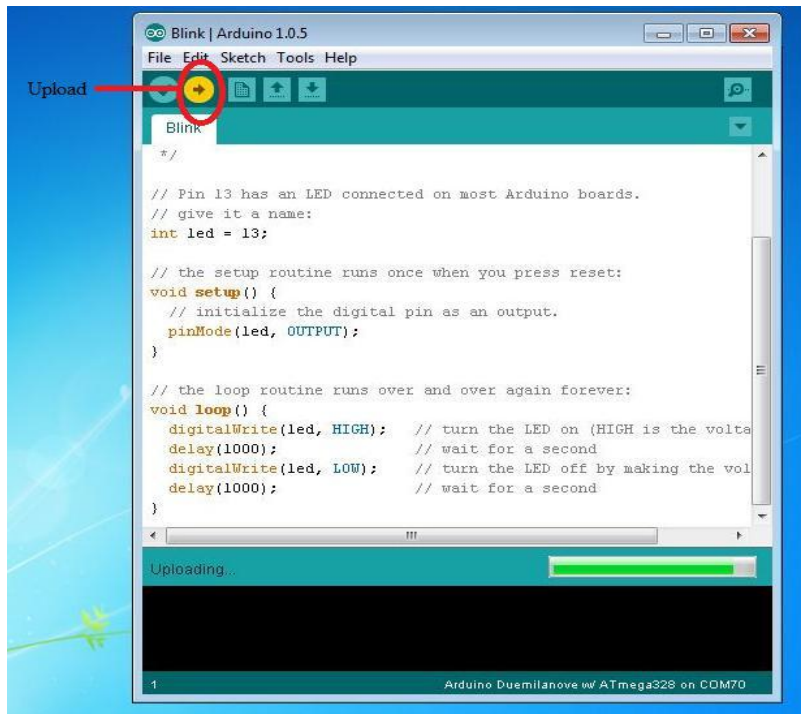

Fig.11. Uploading the program

Step 8:Result.

The final result can be obtained after running this program

\section{Step 9: Observing the Output}

The output can be observed on the serial monitor depending on the given algorithm and instructions.

\section{RESULTS}

The following are the possible corresponding execution states of our circuit:

Without the helmet and acceptable level/ no alcohol consumption.

As soon as both the circuits get powered up, the arduinos on both the ends start executing the respective codes dumped to them. Firstly a radio link communication is established between the transmitter and the receiver sections. The Arduinonano on the transmitter first enables the capacitive switch to detect the presence of the helmet over the head and receives the output input ' 0 ' from it, the Arduino then directly transmits the ' 0 ' bit to the receiver, which eventually means the safety of the rider has been compromised and the operation of the bike must be held off The aduinouno on the receiver end gets the ' 0 ' input and cuts off the power supply to the motor by sensing the data ' 0 ' to the IC from the DIGITAL pin 4.

Thus in the case of the rider not wearing the helmet and not drunk, the MOTOR is either switched to off or remains OFF.

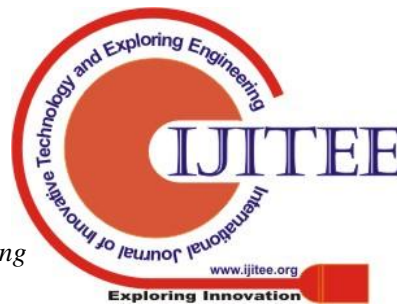


Without the helmet and unacceptable level of alcohol consumed.

Here again as the case is without helmet, the value returned by the capacitive switch is ' 0 ', which eventually puts the operation of MOTOR to OFF, with the back end values and computations being the same as the previous case.

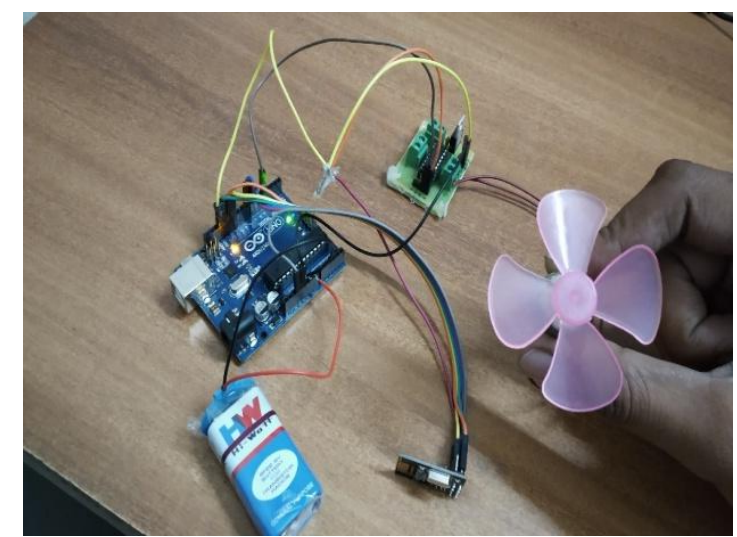

Fig.12. Motor OFF

With the helmet and acceptable or no alcohol consumption.

In this case, as the Arduino enables the capacitive switch, it receives an input ' 1 ', indicating the presence of helmet over the head of the rider, then the Arduinochecks the estimation of $\mathrm{BAC}(\mathrm{Blood}$ Alcohol Content) given by the alcohol sensor, that value is scaled, mapped and then compared with the acceptable threshold BAC level and obtains the result to be below the threshold as it was, then the Arduinotransmitsa bit ' 1 ' to the receiver section, the receiver section turns the MOTOR ON or maintains the MOTOR operation to be ON.

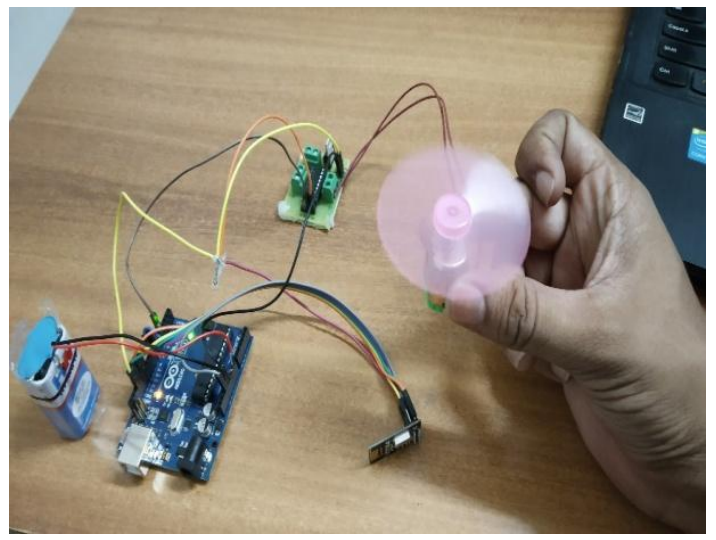

Fig.13. Motor ON

With the helmet and unacceptable the alcohol consumed.

Arduino receives a bit'1' from the capacitive switch and obtains the BAC level from the breath of the person using the alcohol sensor, it compares the value with the threshold and finds that to be beyond the threshold, then the Arduino sends a bit ' 0 ' to the receiver section, which puts the MOTOR to OFF state.

\section{CONCLUSION}

Little more tweaks to this system can make it mandatory in vehicles in such a way that it can be placed in new bs norms because by the end of the day we commute to be more productive, but it doesn't mean that one can risk their life. Riders safe guard can replace the locking system. Riders safeguard 2.0 is used to unlock the vehicles by adding biometric which may generate a floating code in order to ignite engines.

\section{REFERENCES}

1. NiravThakor, TanmayVyas , Divyang Shah "Automatic Vehicle Accident Detection System Based on ARM \&GPS", International Journal for Research in Technological Studies, Vol-1, Issue - 1, pp :17-19, Dec 2013.

2. S.Sonika, Dr.K.Sathiyasekar, S.Jaishree "Intelligent accident identification system using GPS, GSM modem”, International Journal of Advanced Research in Computer and Communication Engineering Vol.3,Issue-2,Feb 2014

3. D. B. Tushara and P. A. H. Vardhini, "Wireless vehicle alert and collision prevention system design using Atmel microcontroller," 2016 International Conference on Electrical, Electronics, and Optimization Techniques (ICEEOT), Chennai, 2016, pp. 2784-2787.

4. K.Murali Chandra Babu, P.A.Harsha Vardhini, "Advanced Vehicle Security System with Spartan FPGA", International Journal of Engineering Research in Electronics and Communication Engineering (IJERECE), pp.86-88, Vol 5 , Issue 4, April 2018.

\section{AUTHORS PROFILE}

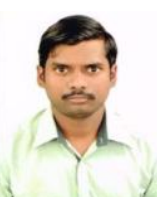

K. Murali Chandra Babu, Assistant Professor in Department of Electronics and Communication Engineering, Vignan Institute of Technology and Science. He published over 17 journals and conference papers including SCOPUS, IEEE and UGC. His research interests cover Low power design, Electronic circuits, VLSI, renewable energy resources and IoT based applications.

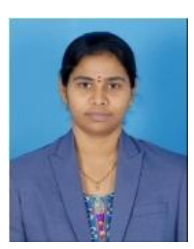

Dr. P.A.HarshaVardhini, professor in the Department of Electronics and Communication, Vignan Institute of Technology and Science, Deshmukhi has 18 years of teaching experience. She pursued her Ph.D in VLSI Design from the department of ECE, JNTUH. Her research interests include Low Power Mixed Signal VLSI Design, FPGA architectures, IoT Embedded Applications \& Wireless Communication. She is an IEEE\& WIE member and life member of IEI, ISOI, IAENG, IAOE. She has more than 90 publications in various journals and conferences at national and International level including SCI, SCOPUS, IEEE, Springer, Elsevier and UGC.

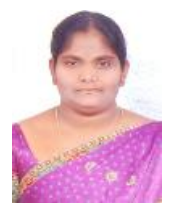

N.Koteswaramma pursuing Ph.D from JNTUA, pursued her M.Tech from Osmania University and B.Tech from JNTUA. Her research interests are in the areas of Wireless Comm, Antenna design, RF circuits, RADAR comm, Image processing. She has 16 journal and conference publications including SCOPUS, IEEE and UGC. 